\title{
Artificial Vision Image Processing Strategy Based on Fuzzy Information Processing
}

\author{
Liping Wang1, Yinhua Wang ${ }^{2}$ \\ ${ }^{1}$ Engineering Technology Research Center of Optoelectronic Technology Appliance. Tongling, Anhui \\ 244000, China \\ ${ }^{2}$ Tongling University, Tongling, Anhui 244000, China
}

Keywords: Fuzzy information processing; Artificial visual images; Image processing

\begin{abstract}
Due to the limited number of microelectrodes implanted into human body in current artificial vision, a large amount of information cannot be transmitted, so the collected images of the external world must be simplified and enhanced to extract the main information in the images. Based on this, this paper proposes a clustering validity method based on fuzzy proximity degree by introducing the global brightness contrast feature of the image and combining the brightness difference features in other color spaces based on fuzzy information processing. By stimulating an undamaged part of the visual pathway, such as retina, optic nerve or visual cortex, through the stimulation electrode array, the blind person can obtain certain visual illusion, thus enabling the blind person to regain some or all of the photosensitive ability. Compared with the traditional clustering validity method, this method has the characteristics of clear concept, simple operation and accurate results.
\end{abstract}

\section{Introduction}

In the past 30 years, human beings have experienced the transition from black and white images to color images. Today, color elements are ubiquitous in our daily life. Retina is a highly complex and fine cell layer in the eyeball. Its function is to convert external light signals into nerve stimulation and transmit it to the next visual pathway [1]. In recent decades, many research teams at home and abroad have launched research on artificial vision replacement schemes, hoping to stimulate visual pathways to generate optical illusion by implanting microelectrode arrays. The development of modern science and technology, the wide application of emerging electronic science and computer technology, and the response progress in biomedical engineering, bionics and other disciplines [2]. For example, at present, the number of electrodes implanted in the visual cortex is still very limited, which can not transmit a large amount of image information. To enable blind people to obtain the same vision as normal people, they must receive a large amount of image information. Bo [3] conducted an experimental study on optic nerve stimulation. They implanted optic nerve electrodes into a blind patient with retinitis pigmentosa and connected the nerve stimulator to output via radio. Based on the fuzzy information inherent in the image, this paper proposes some new image processing methods using fuzzy information processing technology, which have achieved good results.

\section{Image Processing Method}

In the research of image processing methods applied to artificial vision, in order to obtain simple and effective image information, real-time image information is obtained through an image acquisition system, and redundant information is removed through an image preprocessing process. An image always has a certain subject. Analyzing and identifying the subject in the image, and then guiding the next action, is the mainstream of human visual activities [4]. The rods are thin and long, feeling extremely weak light but unable to distinguish colors. Retinal cone cells are thick and short. They only react to strong light and can feel different colors. Under different weights, they are superimposed to obtain the final saliency map. Finally, low pixelation processing is carried out, and 
low-resolution visual perception is simulated. The camera can be aimed at a marker with a plurality of independent markable points, images are collected on the marker through different angles and distances, and the parameters of the camera are calculated by using images of different angles and distances. Different image processing strategies can be used to reduce the amount of computation. When a simpler processing strategy cannot achieve better results, the processing system should automatically switch to a more complicated processing strategy at the next higher level.

If the average gray scale of an image is 100 , the average gray scale of the background is 90 , the standard mean square deviation of the target is 10 , and the standard mean square deviation of the background is 10 . Smoothing of degraded images is achieved by minimizing the cost function, which is [5]:

$$
C=\sum_{u \in U}\left\{x(u)-f(u)^{2}+\lambda \sum_{v \in y(u)} x(u)-x(v)^{2}\right\}
$$

$f(u)$ represents the gray value of the degraded image at $u=(i, j) ; u$ represents the set of pixels; $x(u)$ represents the approximate value of the gray value of the image at $u=(i, j)$ before degradation. In this section, we select the eight neighboring pixels, the size is $3 * 3$, the pixel with the center at $(i, j)$ out of the window $\lambda$ is 1 , and the formula of the cost function is transformed into the following formula:

$$
(1+7 \lambda) x(u)=f(u)-\lambda \sum_{v \in y(u)} x(v)=0
$$

Each pixel in the image has its own pixel value, which determines the final display of the image. By averaging the pixel values in the image. It is also divided into two types, one is to perform pointby-point operations on images, and the other is to perform operations on the spatial domain related to processing the neighborhood of image points, called local operations. Then the image cannot be segmented using the general threshold segmentation method, so the image preprocessing is realized by minimizing the cost function, and then the image is further processed using fuzzy entropy [6]. The simplified image is used to generate binary image by adaptive thresholding method, and then the image is extracted with information. Four-direction edge extraction algorithm is used for information extraction. The visual restoration of artificial vision does not completely restore and present every detail in the environment, such as color, depth, texture and other information, which is beyond the scope of artificial vision restoration at this stage. At the same time, it should be able to be implanted stably, with certain elasticity but not too much suppression or involving of nerve tissue. There is sufficient energy supply to drive the stimulation electrode array, but the generated heat will not cause damage to peripheral functional tissues.

Fuzzy sets have different representation methods, which can be expressed as

$$
\bar{C}=\left\{\left(y, \eta_{\bar{C}}(y)\right), y \in Y\right\}(3)
$$

Where $Y$ is the universe and $\bar{C}$ is a fuzzy set in the universe.

If $Y$ is a finite set, $\bar{C}$ can be expressed as

$$
\bar{C}=\sum_{K} \eta_{\bar{C}}\left(y_{i}\right) / y_{i} \quad\left(y_{i} \in Y\right)
$$

If $Y$ is an infinite set, $\bar{C}$ can be expressed as

$$
\bar{C}=\int_{Y} \eta_{\bar{C}}(y) / y \quad(y \in Y)
$$

Levin algorithm treats the colorization of black and white images as a global optimization 
problem. The algorithm is based on the basic assumption that pixels with the same gray value should have similar colors [7]. The existing images are recombined into ideal images; Then the recombined image is thresholded. It is often necessary to convert the image originally defined in the pixel space into other spaces in some form, and make use of the special properties in these spaces to make certain processing conveniently, and finally convert into the image space to obtain the desired effect. Adjust the pixel color value according to gaussian curve, that is, Gaussian blur can count the pixel color values around a certain point according to gaussian curve, and obtain the color value of this curve by using the mathematical weighted average calculation method. For this reason, some areas of the black-and-white image are coated with appropriate colors as guides or restrictions for colorization. The final result of colorization is to keep the color of the coated pixels unchanged. Then, regardless of the complexity of the image, edge extraction is required to obtain edge contour information, and mathematical morphology algorithm expansion and erosion operations are selected for image enhancement processing according to different complexity. Then try to transform its shape features or texture features into the required form in the pattern recognition method.

\section{Result Analysis}

In order to prove the advantages of colorization algorithm based on Bayesian non-local mean inference, we will compare it with the most representative colorization algorithm based on manual intervention, including Levin colorization algorithm [8] and Yatiz colorization algorithm based on geodetic distance [9]. Due to the influence of external ambient light, the dynamic range of brightness in the images collected by the equipment is small. Histogram equalization is an effective image enhancement method based on gray mapping transformation in spatial domain. There is a delay in the occurrence time of the optical illusion point, and the topological relation between the electrode array and the optical illusion point position is different for different individuals. Under the given method and resolution, if the difference between the identification rate of the test individual and the standard deviation of the whole identification rate is more than two times, the individual's data will be considered unreliable and will be deleted from the overall data. The visual perception after retinal prosthesis implantation is simulated through low pixel processing. According to epidemiological data, this study uses data sets of 50 objects familiar to blind people in their daily life to test the algorithm. According to the synchronous oscillation characteristic of visual cortex, namely, the pulse emission caused by the excitation of visual cortex neurons can cause the excitation and pulse emission of a plurality of neurons in neighboring similar states, and can be applied to a neuron group acting on the same stimulus. Then the pixel difference between the original image to be tested and the blurred image is calculated, the distribution of the pixel difference is counted to detect the blurred trace, and the blurred tampered area is located according to the pixel difference.

The image stimulation of visual cortical nerve cells to the external world can be expressed in the form of receptive fields, while the sparsely coded basis functions have similar properties to receptive fields of simple cells. The signal-to-noise ratio and the specific gravity of non-zero coefficients of reconstructed images under different thresholds are shown in Table 1. It can be seen that since the basis functions of sparse coding are not orthogonal, sparse coding cannot completely restore the original image without compressing sparse coefficients.

Table 1 Signal-to-noise ratio and proportion of non-zero coefficients of reconstructed images under different thresholds

\begin{tabular}{ccc}
\hline Threshold & Signal to noise ratio(dB) & Non-zero coefficient ratio \\
\hline 1 & 15.36 & 2 \\
\hline 0.3 & 14.21 & 0.25 \\
\hline 2 & 15.02 & 0.07 \\
\hline 0.1 & 15.07 & 0.14 \\
\hline
\end{tabular}

In the image enhancement method, if some human visual characteristics are introduced, the effect will be significantly improved. However, most existing algorithms do not introduce human 
visual characteristics. Through the principle of minimum fuzzy entropy to realize the processing of pixel gray value, then the gray value is 100 , which meets the actual needs. Obviously, the colorization algorithm based on Bayesian non-local mean inference gives more strict spatial structural constraints through the non-local likelihood probability model and Gaussian MRF prior probability model, thus effectively avoiding the false diffusion of color. After histogram equalization, the gray level of the image is almost evenly distributed throughout the dynamic interval. Histogram equalization is a gray mapping transformation based on spatial domain technology. Its meaning is to make the number of pixels distributed on each gray level equal or basically equal through processing. Although pixelated images cannot provide enough information, some subjects can still distinguish correctly. Color images expressed in different color spaces also have different characteristics. Whether the color space is selected properly has an important influence on the processing effect of color images. The number of neuron responses far from zero value is small, that is, the number of neurons that generate strong responses is small, which accords with the sparse response characteristics of the visual system. Therefore, the peak-valley characteristic of the histogram is enhanced, and a smooth image under the filter bank is obtained.

The subjects can identify some objects with complex appearance characteristics at the resolution, but they cannot correctly identify some objects with relatively simple appearance, such as a bulb or a banana. Figure 1 shows the average recognition rate of two objects with significant shape differences, so as to study the influence of shape features on recognition near the recognition threshold.

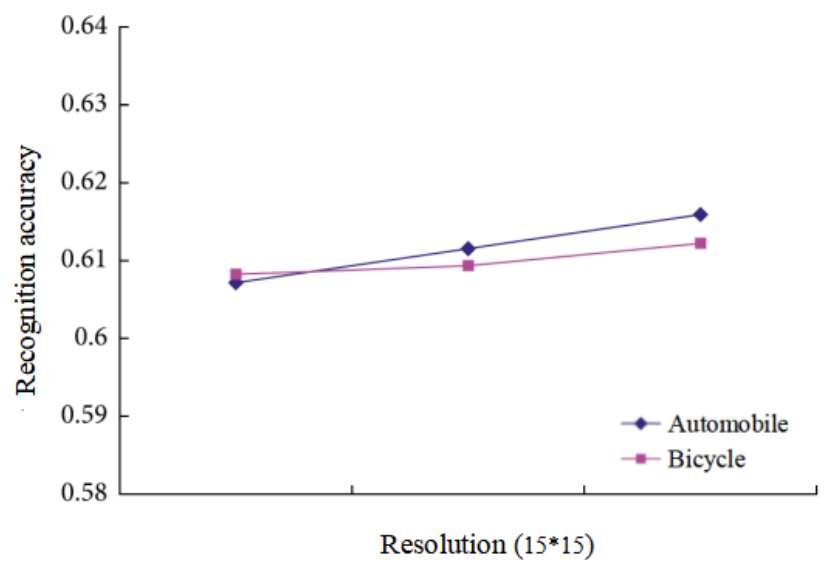

Figure 1 Comparison of average recognition rates of 2 different image modes under $15 * 15$

The frequency of pulse train stimulation has two effects on the optical illusion point. First of all, a series of pulse sequences must reach above the critical flicker frequency in order for the blind to have a continuous sense of light perception, otherwise the pulse train stimulation will become continuous flicker of many points instead of continuous lighting of a fixed point. The increase of stimulation frequency will reduce the threshold current (fig. 2) that causes photophobia, which will increase the brightness of photophobia under other same stimulation parameters.

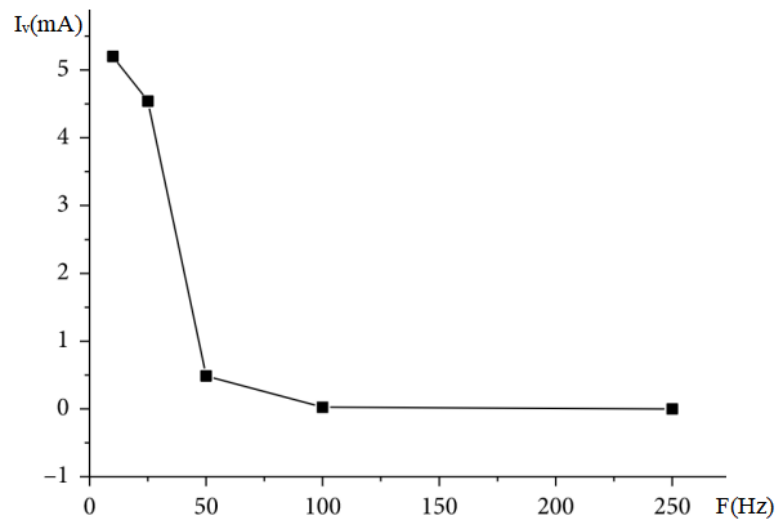

Figure 2 Relation curve between threshold current and stimulation frequency 
Neurons do not respond equally to external input information, but respond to some input information that they consider important. The experimental results show that these processes not only filter the background noise caused by foreground enhancement, but also make up for the lack of foreground information compared with the original image, realizing background suppression and extracting foreground better. When the number of pixels increases to $15^{*} 15$, the recognition rate of the image patterns obtained by the two kinds of image processing strategies is significantly different. The strategy of binarizing relative edges shows better effect, while the influence of pixel shape is relatively small. If the histogram distribution is concentrated, it is considered that the gray distribution of the image is uneven. At this time, histogram equalization is performed on the image to improve the contrast of the image. Such as identifying objects in an image. The difference between it and image processing is that the image obtained through image processing such as enhancement, compression, reconstruction and repair is still an image. The traditional colorization and redrawing methods cannot colorize the pixels in black-and-white images that have lost information effectively. However, the combined restoration and colorization algorithms can obtain better visual effects in colorizing the missing information areas of the images. According to this principle, human eyes are more sensitive to noise in the gentle part of the image than in the detailed part. This method can effectively reduce the error rate in the process of image segmentation and improve the visual effect of image segmentation.

\section{Conclusion}

Image segmentation is the basis of image understanding, analysis and pattern recognition. It plays an important role in image processing and is widely used in real life. This paper proposes a simple and effective fuzzy detection method. The fuzzy function is mainly used to perform a fuzzy process on the image to be tested, and then the pixel difference between the original image to be tested and the blurred image is calculated. On the basis of obtaining the foreground object and background information, two image expression strategies are proposed, i.e. gray separation, low pixelation and background marginalization. The core of the two image expression strategies is to enhance the contrast between the foreground and background information. According to the parallax, the background information of the image and the information of the objects close to the blind are removed, thus reducing the information content of the image and transmitting the most important image information with a small number of microelectrodes. For the colorization of damaged images, the experimental results show that the algorithm is superior to the typical colorization algorithm. Compared with the repair+colorization method, the experimental results show that our algorithm has an advantage in computation time.

\section{Acknowledgements}

Tongling university talent research start-up fund project "research on photoelectric detection technology of circuit board surface defects based on image registration" (2015tlxyrc02); Anhui University Natural Science Research Project "Research and Application of Face Image Recognition Technology" (KJ2017A472).

\section{References}

[1] Guo Yuanrong. Development and application of image processing and recognition technology. Electronic technology and software engineering, no. 1, pp. 58-59, 2018.

[2] Xu Zhiyan. Research progress of Argus II artificial retinal system. Chinese Journal of Experimental Ophthalmology, vol. 37, no. 2, pp. 157-160, 2019.

[3] Bai Taotao. Research and application of IC shallow insertion detection based on machine vision and image processing. Journal of Hebei North University (NATURAL SCIENCE EDITION), no. 4, pp. 36-40,2015. 
[4] Li L, Xue J, He X, et al. [Assessment of skin aging grading based on computer vision][J]. Sheng Wu Yi Xue Gong Cheng Xue Za Zhi, vol. 34, no. 3, pp. 449-455. 2017.

[5] Wang Shasha. Application of artificial intelligence in eye image diagnosis. Chinese Journal of Experimental Ophthalmology, vol. 36, no. 10, pp. 796-799, 2018.

[6] Wu Hongyun, Wei Xi. Application of machine vision technology in ice breaking. Radio communication technology, vol. 41, no. 1, pp. 84-86, 2015.

[7] Jia Honglei, Wang Gang, Guo Mingzhuo, et al. Method and experiment of corn plant quantity acquisition based on machine vision. Journal of agricultural engineering, vol. 31, no. 3, pp. 215-220, 2015.

[8] Tang Bo, Kong Jianyi, Wu Shiqian. Overview of machine vision surface defect detection. Chinese Journal of image graphics, vol. 22, no. 12, pp. 1640-1663, 2019.

[9] Zhang Ting, Huang Chang, Xiao Yujie. Algorithm improvement of feature point extraction based on SIFT. Information communication, no. 2, pp. 66-67, 2016. 